\title{
Imaging motile pathogens by light microscopy and cryo electron tomography
}

\author{
F. Frischknecht,* S. Munter,* M. Kudryashev,* S. Lepper, * S. Hegge,* W. Baumeister, ${ }^{* *}$ Reinhard \\ Wallich, ${ }^{* * *}$ U.S. Schwarz, ${ }^{* * * *}$ M. Cyrklaff*
}

* Department of Parasitology, Hygiene Institute of the University of Heidelberg Medical School, Im Neuenheimer Feld 324, 69120 Heidelberg, Germany

** Department of Molecular Structural Biology, Max Planck Institute for Biocheistry, Am Klopferspitz 18, 82152 Martinsried, Germany

*** Institute for Immunology, University of Heidelberg Medical School, Im Neuenheimer Feld 305, 69120 Heidelberg. Germany

**** BIOQUANT, Center for Modelling and Simulation in the Biosciences, University of Heidelberg, Im Neuenheimer Feld 267, 69120 Heidelberg, Germany and Institute of Zoology, Theoretical Biophysics Group, University of Karlsruhe, Kaiserstrasse 13, 76131 Karlsruhe, Germany

We are interested in understanding the motile behavior of pathogens and currently work on two arthropod-borne disease agents: Plasmodium, the causative agents of malaria and Borrelia, the causative agents of Lyme disease.

Plasmodium undergoes a complex life cycle in its vertebrate host and mosquito vector. We examine Plasmodium sporozoites, the stages that are transmitted during a mosquito bite. Sporozoites are ejected with the mosquito saliva [1] into the skin of the host. There they move rapidly and can enter blood capillaries $[2,3]$. Sporozoites eventually invade hepatocytes to further differentiate into red blood cell invading parasite forms that ultimately cause the disease. We use a number of light microscopy approaches including reflection interference contrast and traction force microscopy to examine the molecular and biophysical basis of motility in vitro, where sporozoites glide in circles as shown in Fig. 1. We also utilize cryo electron tomography to investigate sporozoites [4]. Sporozoites had to evolve to be less than 1 micrometer thick so they can still pass through the narrow salivary ducts of the mosquito and can thus be imaged by electron microscopy without the need for sectioning. Our latest results will be presented for both imaging approaches.

Borrelia are spirochetes that are transmitted into the skin during the bite of an infected tick. Different species of Borrelia cause different symptoms of Lyme borreliosis. We undertake both quantitative light microscopy imaging as well as cryo electron tomography to learn more about these emerging pathogens with cause around 80.000 annual cases of infection in Germany alone. A comparative tomography study of the three main borreliose species B. burgdorgeri, B. garinii and $B$. afzelii revealed an overall similar architecture and showed a number of previously little or not recognized details, including the presence of cytoplasmic vesicles at the site of bacterial cell division [5]. Two poles of different Borrelia species are shown in Fig. 2. We further found evidence for a potentially new mechanism of horizontal gene exchange between Borrelia that could explain the large genomic diversity between strains [6].

References

[1] F. Frischknecht et al., Cell. Microbiol. 8 (2004) 687.

[2] R. Amino et al., Nat. Med. 12 (2006) 220. 
[3] R. Amino et al., in S.L. Shorte and F. Frischknecht (eds.) Imaging Cellular and Molecular Biological Functions, Springer, Heidelberg, 2007.

[4] M. Cyrklaff et al., J. Exp. Med. 204 (2007) 1281.

[5] M. Kudryashev et al., Mol. Microbiol. 71 (2009) doi: 10.1111/j.1365-2958.2009.06613.

[6] This research was supported by the Federal German Ministry of Education and Science (BMBF - Biofuture), the German Research Foundation (DFG, SFB 544 and SPP 1128) and the University of Heidelberg Frontier Program. FF is an affilitated member of the European Network of Excellence BioMalPar.
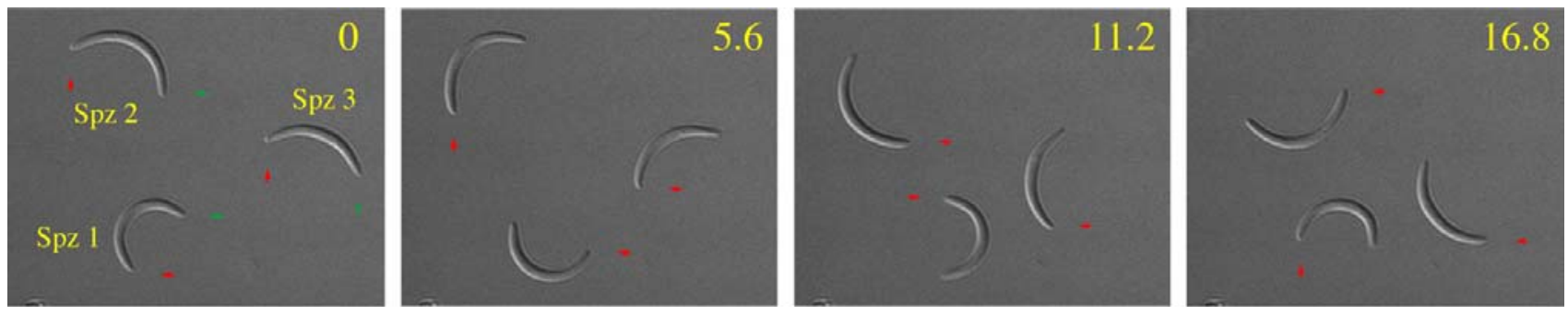

FIG. 1. Differential interference contrast time-lapse images of sporozoites from the rodent malaria parasite Plasmodium berghei. Time is indicated in seconds. Red arrowheads indicate the position of the front end of each parasite at each frame. Green arrowheads indicate the rear end in the first frame.

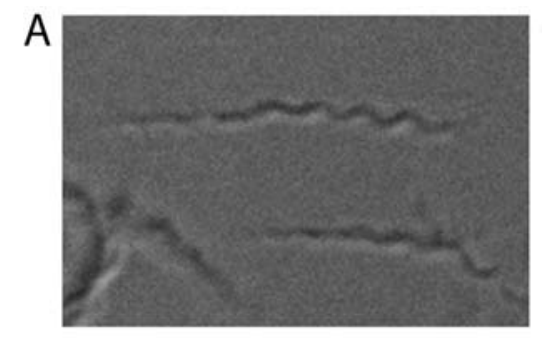

B
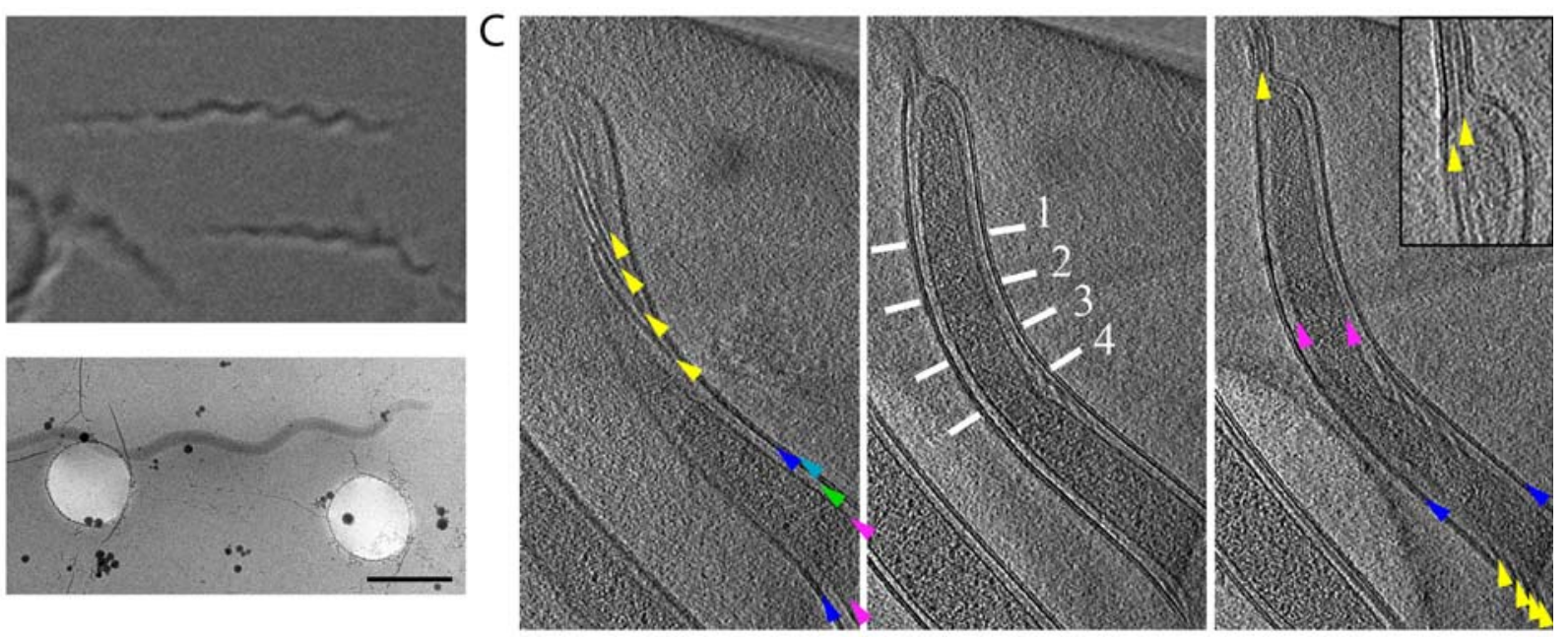

FIG. 2. Borrelia burgdorferi: A: phase contrast image. B: Cryo electron micrograph. C: Three sections from a tomogram with arrowheads indicating periplasmic flagellar filaments (yellow), outer membrane sheath (blue), peptidoglycan (green) cytoplasmic membrane (magenta). From reference 5. Copyright: the authors. 